\title{
Ferromagnetic Resonance Controlled by Micro-regions in Fe-Co Based Thin Film
}

\author{
Xiaojia Luo, Peiheng Zhou ${ }^{\mathrm{a}}$, Xin Wang, Jianliang Xie and Longjiang Deng \\ ${ }^{1}$ State Key Laboratory of Electronic Thin Films and Integrated Devices, University of Electronic \\ Science and Technology of China, Chengdu 610054, China; \\ ${ }^{2}$ National Engineering Research Center of Electromagnetic Radiation Control Materials, University \\ of Electronic Science and Technology of China, Chengdu 610054, China. \\ azhouph1981@163.com
}

Keywords: Magnetodynamics, .Anisotropy, Antidots, Permeability.

\begin{abstract}
FeCoBSi amorphous thin films containing circular antidots are fabricated on the silicon substrate by photolithography, DC magnetron sputtering and lift-off patterning. The films are divided into several micro-regions by antidots which have different demagnetizing field. The magnetic properties of these thin films are studied by measurement of the dynamic magnetic spectrum and hysteresis loop. The demagnetizing field in micro-regions deteriorates macroscopic uniaxial magnetic anisotropy (UMA). Diverse resonance peaks are found for various antidots. The dipolar coupling among micro-regions and the natural resonance independent part of micro-regions determine the dynamic magnetic spectrum together. The mechanisms of ferromagnetic resonance are proposed to be related to the special domain structure caused by the demagnetizing field in corresponding micro-regions. In order to study the configuration of magnetic moments in micro-regions, the object oriented micromagnetic framework (OOMMF) is used to simulate the dynamic magnetization process.
\end{abstract}

\section{Introduction}

Ferromagnetic thin films with periodic arrangements of holes, so called antidote lattices, have been broadly investigated in the last decade [1-3]. Patterning holes into ferromagnetic thin films is a simple and effective way to engineer magnetic properties. Since antidots can reshape the demagnetizing fields in thin-film structures and pin domain walls, the anisotropies [4, 5], switching characteristics [6], coercivities [7], and remanences [8] of ferromagnetic thin films can be adjusted.

The anistropies in as-deposited amorphous films mainly include shape anisotropy caused by antidots, and induced anisotropy caused during fabrication. Shape anisotropy mainly works in regions where the magnetization remains uniform and rotates coherently [9]. Therefore, one of the precondition to efficiently control the anisotropy field by micro-region is to prevent the formation of multidomain in the thickness direction of the film.

It is known that the FMR is determined by saturation magnetization $\left(M_{\mathrm{s}}\right)$, anisotropic field $\left(H_{\mathrm{e}}\right)$, and damping factor $(\alpha)$. For continuous FeCoBSi amorphous films, there is usually only one FMR peak in $0.5 \mathrm{GHz} \sim 12 \mathrm{GHz}$ caused by its own material characteristic. In the past decade, researchers have tried various methods to control the factors mentioned above to achieve the required FMR performances [10-12]. Various spin wave modes have been discovered in patterned films and designated as the reason of multi-peak resonance. Studies of spin dynamics in positive dots and wire structures have shown that spin wave will be confined by lateral boundaries [13] and domain walls [14]. In amorphous thin films, magnetic anisotropy will not be affected by crystal structure, so the main contribution to construct magnetic domains comes from antidots and other induced characteristics.

In our previous work [15], the effect of rectanglar antidots on resonance frequency of thin film has been analyzed. In this work, we discuss the relationship between introduced anisotropy and shape anisotropy. Films with different kinds of antidots are prepared to prove the validity of the analyze 
method we have proposed, which provides a way to design the antidots patterns in ferromagnetic amorphous thin film.

\section{Experiment}

$\mathrm{Fe}_{66} \mathrm{Co}_{17} \mathrm{~B}_{16} \mathrm{Si}_{1}$ amorphous thin films of circular antidots lattices respectively were fabricated on the silicon substrate by photolithography, DC magnetron sputtering and lift-off patterning. The size of antidots and distance between them are shown in Fig. 1. In the sputtering process, the base pressure was less than $5 \times 10^{-4} \mathrm{~Pa}$, the sputtering voltage was $0.3 \mathrm{kV}$, and the sputtering current was $0.15 \mathrm{~A}$. A magnetic field $\left(H_{a}\right)$ of $1.2 \times 10^{6} \mathrm{~A} / \mathrm{m}$ was applied during the sputtering process to induce an in-plane uniaxial anisotropy.

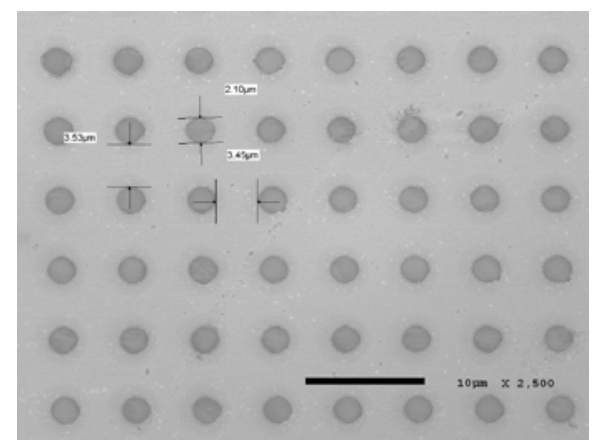

Fig. 1. The SEM photo of films containing circular antidots with antidots diameter $2 \mu \mathrm{m}$ and distance $3.5 \mu \mathrm{m}$.

The morphology of the films was observed by scanning electron microscope (SEM). The microwave permeabilities of these films in remanent state were obtained by vector network analyzer (VNA) using shorted microstrip-line method without bias field. In the test process, the perturbation field $\boldsymbol{h}$ goes along the $x$-axis. To measure the hysteresis loops of in-plane easy and hard axis of these films by vibrating sample magnetometer (VSM), the samples were cut into $5 \mathrm{~mm} \times 5 \mathrm{~mm}$ pieces.

\section{Results and discussion}

In continuous amorphous FeCoB films, the applied field in depositing process will introduce an induced anisotropy which makes the continuous film possessing an UMA character [16]. However, when antidots are introduced into the films, the macroscopic UMA is destroyed. We had indicated there is no obviously differences between the hysteresis loops tested at two directions (easy axis and hard axis) of the rectangular samples. It is illustrated that the macroscopic UMA is no longer existent. However, during deposition, the applied field has affected the atomic arrangement. In order to verify the influence of induced anisotropy, the hysteresis loops of circular antidots samples are measured by VSM, and shown in Fig. 2. In the two measured directions, there is no shape anisotropy. The remanence of easy axis is larger than hard axis obviously. Therefore, the growth trend of atom introduced by applied field in depositing process is still existing. Which means in micro-region, the effective anisotropy maybe still formed by the introduced anisotropy and shape anisotropy.
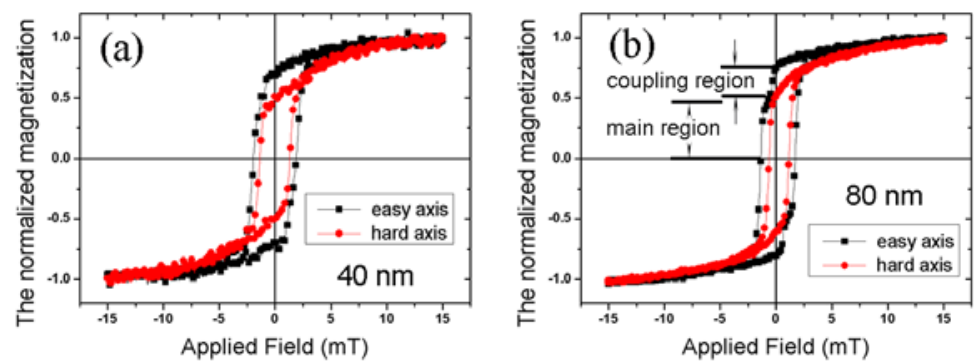

Fig. 2 The hysteresis loops of 40 nm-thick (a) and 80 nm-thick (b) circular antidots samples. The easy axis is along the direction of $H_{a}$, and the hard axis is perpendicular to the easy axis. 
From Fig.2, we can find in each hysteresis loop of easy axis, there is a step in the second and forth quadrant. This step is obvious in the $80 \mathrm{~nm}$ sample especially. This phenomenon indicates that there are two regions with the different difficulties of magnetization. The coupling region and main region are corresponding to the region in Fig.3. The coupling region is about $1 / 2$ of the main region. Due to the demagnetizing field, the coercivity of the coupling region is less than the main region.

In order to understand the configuration of magnetic moments in films with antidots under the perturbation field, OOMMF is used to simulate the dynamic magnetization. The periodic boundary condition 2D_pbc module was introduced to mimic the infinite-size state in two in-plane dimensions. The dynamic magnetization process of films with different antidots are simulated at their measured resonance frequency. The films are magnetized to saturation along $y$ axis as the initial state. A perturbation field with $0.006 \mathrm{mT}$ amplitude is applied along the $x$-axis. The exchange constant is set as $1 \times 10^{-11} \mathrm{~J} \cdot \mathrm{m}^{-1}$. The saturation magnetization is $1.5 \times 10^{6} \mathrm{~A} \cdot \mathrm{m}^{-1}$. The $\mathrm{x}-\mathrm{y}$ cell size is $10 \mathrm{~nm} \times 10 \mathrm{~nm}$ and z cell size is $10 \mathrm{~nm}$. The uniaxial anisotropy energy is considered to be $2000 \mathrm{~J} \cdot \mathrm{m}^{-3}$ [17]. In Fig. 3, the image shows that simulated model is consisting of 4 units. For films with same composition, their saturation magnetizations can be considered as the same. So the differences of dynamic magnetic properties for our samples are mainly caused by anisotropy field. In order to analyze the anisotropy, the films with antidots are divided into two micro-regions. The micro-regions are marked in Fig. 3.

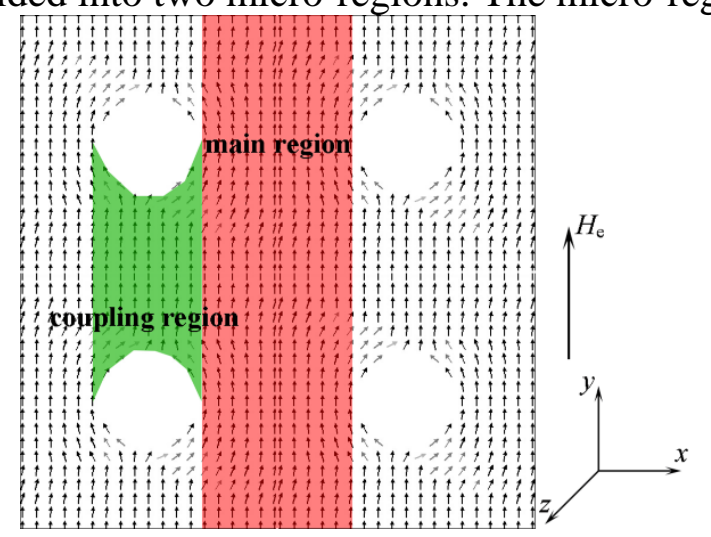

Fig. 3 The configuration of magnetic moments in micro-regions got by OOMMF for the film of circular antidots at $5.25 \mathrm{GHz}$. The micro-regions are determined by the demagnetizing field and the magnetic moment configuration.

It is found that the configuration of magnetic moments is periodic and regional. The magnetic moments in thin film with circular antidots are inclined towards the direction of $H_{a}$, which is close to the streak structure in thin film. Then, the film with circular antidots is divided into coupling region and main region (the region with the aligned configuration of magnetic moment). The antidots shape-dependent demagnetizing energy plays an important role. Bedanta and Mallick have certified the effect of some kinds of antidot on domains by experiment which is related to the effective anisotropy field [18].

On account of the effect of shape anisotropy and introduced anisotropy, the effect anisotropy will diverge from the induced easy axis. In order to analyze this effect, the complex permeability related to saturation magnetization and effective anisotropy field can be gotten by equation (1) [19],

$$
\mu_{i}=1+\frac{\gamma M_{s}\left[\gamma H_{e}+\left(N_{z}-N_{y}\right) \gamma M_{s}\right]+i \alpha f / 2 \pi}{\left\{\left[\gamma H_{e}+\left(N_{x}-N_{y}\right) M_{s}\right]+i \alpha f / 2 \pi\right\}\left\{\left[\left(\gamma H_{e}+\left(N_{z}-N_{y}\right) \gamma M_{s}\right]+i \alpha f / 2 \pi\right\}-(f / 2 \pi)^{2}\right.},
$$

where $\gamma$ is the gyromagnetic ratio considered to be $2.8 \times 10^{9} \mathrm{~Hz} / \mathrm{T}, \omega$ is the angular frequency, and $H_{\mathrm{e}}$ is effective field. Moreover, $N_{\mathrm{x}}, N_{\mathrm{y}}$, and $N_{\mathrm{z}}$ are on behalf of the demagnetization factors along each axial direction corresponding to the coordinate shown in Fig. 4 respectively. According to the hypothesis of equation (1), the magnetic moments are assumed to align in the direction perpendicular to $\boldsymbol{h}$ (along with the $y$-axis). In measuring process, the film was treated as an unbroken uniform material. Hence, the measured permeability should be proportional to the superficial area percentage of the aligned magnetic moments in each region. For example, according to the volume percentage of 
the main region in Fig.3, the measured permeability value is about $40 \%(2 \mu \mathrm{m} / 5.5 \mu \mathrm{m})$ of its' theoretical value, on the assumption that there is no coupling.

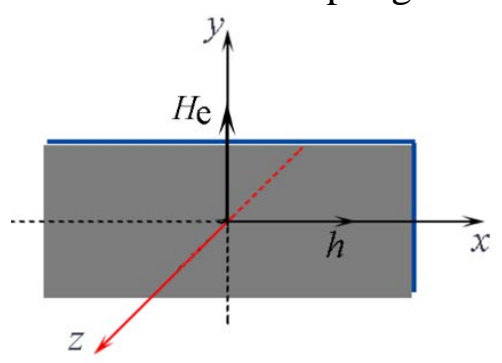

Fig. 4 The sketch map of film in the coordinate system. The plane of film is in the $x, y$ plane, and the thickness direction is along the $z$-axis.

Then, the numerical values of $M_{\mathrm{s}}=1.5 \times 10^{6} \mathrm{~A} \cdot \mathrm{m}^{-1}$ and $H_{\mathrm{e}}=3200 \mathrm{~A} \cdot \mathrm{m}^{-1}$ in continuous film are treated as the effective value in films with antidots. And, the demagnetization factors can be obtained by [20]

$$
\left\{\begin{array}{l}
N_{x}=\frac{2}{\pi} \operatorname{tg}^{-1} \frac{b \sqrt{a^{2}+b^{2}+c^{2}}}{a c} \\
N_{z}=\frac{2}{\pi} \operatorname{tg}^{-1} \frac{a \sqrt{a^{2}+b^{2}+c^{2}}}{b c} \\
N_{z}=1-N_{x}-N_{y}
\end{array},\right.
$$

where $a, b, c$ stand for the side length along the $x, y, z$-axis respectively. According to equation (1) and (2), the resonant frequency of micro-region can be calculated.
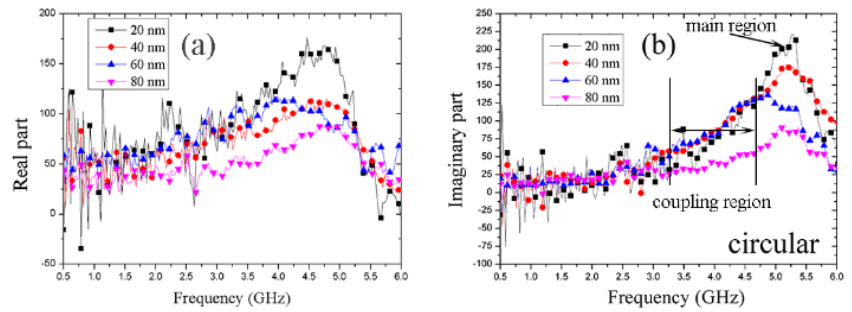

Fig. 5 The permeability of films with circular antidots measured by VNA. (a) Real part of the permeability; (b) Imaginary part of the permeability.

The measured permeabilities of films with circular antidots are shown in Fig. 5. After the continuous films are separated by antidots, the positions of resonance peaks hardly move with the thicknesses of films. Only one broad peak is presented in Fig. 4 (b). This phenomenon is consistent to our analysis in Fig. 3. The imaginary part permeabilities of our films with circular antidots are similar to the results of films with streak patterns in Ref. [21]. It can be inferred that the configuration of magnetic moments (domain structure) is one of the determinant factor of dynamic magnetic properties.

Although the regions have been divided as shown in Fig. 3, their shape factors are different. In this case, the $40 \mathrm{~nm}$-thick film is used as an example to describe the shape factors with length of $a, b, c$ as listed in Table 1. In accordance with specific conditions, the length along with $y$-axis of the main regions is treated as infinite. Because $b$ is the same with the width of the thin film which is equal to 5 $\mathrm{mm}$, that much larger than $a$ and $c$.

Table 1 The side length and demagnetizing factors of themain region in $40 \mathrm{~nm}$ film with circular antidots.

\begin{tabular}{ccccccc}
\hline & \multicolumn{3}{c}{ Length $(\mathrm{nm})$} & \multicolumn{3}{c}{ Demagnetization factor } \\
\cline { 2 - 7 } & $a$ & $b$ & $c$ & $N_{x}$ & $N_{y}$ & $N_{z}$ \\
\hline Main region & 350 & 40 & $\infty$ & 0.0073 & 0 & 0.9927 \\
& 0 & & & & & \\
\hline
\end{tabular}


In previously work, we has investigated the thcknesses of thin films with rectangular rarely affect the resonance frequencies. According to equation (1) and (2), the resonance frequencies should increase with the thicknesses. But, the resonance frequencies almost stay the same, because the anisotropy field is reduced by the dipole coupling between regions.

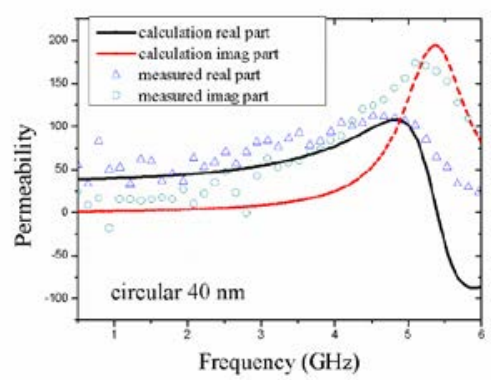

Fig. 6 The fitting result of 40 nm-thick film with circular antidots.

By bringing the values of Table 1 into equation (1), the fitting result of the main region is gotten and shown in Fig. 6. The amplitude is multiplied by 0.4 for its superficial area percentage. Since the regions are closely connected, coupling probably exists among them. The coupling makes the resonant frequency of each region offset. According to equation (1), when the direction of magnetic moments is parallel to $H_{\mathrm{e}}$ (perpendicular to perturbation field $\boldsymbol{h}$ ), the position of resonant frequency is the highest. This is why the coupling regions are all on the left side of main region in Fig. 5(b).

\section{Summary}

Diverse resonance peaks are presented for different antidots in ferromagnetic thin film. The dynamic magnetic spectrum of these ferromagnetic films is influenced by dipolar coupling among micro-regions and natural resonance in the independent part of micro-regions. The mechanisms of ferromagnetic resonance are proposed to be related to the special domain structure what is caused by the demagnetizing field in micro-region. In these micro-regions, shape anisotropy is the main influence factor, and induced anisotropy plays a supplementary role. Based on these analyses, FMR can be controlled by micro-region in amorphous thin films.

\section{Acknowledgment}

The authors are grateful to the supports from the National Natural Science Foundation of China under Grant Nos. 51025208 and 61001026, the Program for Changjiang Scholars and Innovative Research Team in University and the Program for New Century Excellent Talents in University (NCET).

\section{References}

[1] C. Castán-Guerrero, J. Herrero-Albillos, J. Bartolomé, F. Bartolomé, L. A. Rodríguez, C. Magén, and L. M. García, Physical Review B, 89 (2014) 144405.

[2] H. Fang, B. Caballero, E. M. Akinoglu, E. Papaioannou, A. García-Martín, J. C. Cuevas, ... and P. Fumagalli, Applied Physics Letters, 106(15) (2015) 153104.

[3] J. Gräfe, M. Weigand, N. Träger, G. Schütz, E. J. Goering, M. Skripnik, ... and U. Wiedwald, Physical Review B, 93(10) (2016) 104421.

[4] W. J. Gong, W. Liu, J. N. Feng, D. S. Kim, C. J. Choi, and Z. D. Zhang, Journal of Applied Physics, 115 (2014) 133909.

[5] C. T. Yu, H. Jiang, L. Shen, P. J. Flanders, and G. J. Mankey, Journal of Applied Physics, 87 (2000) 6322-6324. 
[6] P. Vavassori, G. Gubbiotti, G. Zangari, C. T. Yu, H. Yin, H. Jiang, and G. J. Mankey, Journal of Applied Physics, 91 (2002) 7992-7994.

[7] L. J. Heyderman, F. Nolting, D. Backes, S. Czekaj, L. Lopez-Diaz, M. Kläui, and P. Fischer, Physical Review B, 73 (2006) 214429.

[8] A. O. Adeyeye, J. A. C. Bland, and C. Daboo, Applied Physics Letters, 70 (1997) 3164-3166.

[9] R. Skomski, J. M. D. Coey, Scripta Materialia, 112 (2016) 3-8.

[10] A. Y. Toporov, R. M. Langford, A. K. Petford-Long, Applied Physics Letters, 77 (2000) 3063-3065.

[11] X. Liu, W. Zhang, M. J. Carter, G. Xiao, Journal of Applied Physics, 110 (2011) 033910.

[12] A. El-Ghazaly, R. M. White, S. X. Wang, Journal of Applied Physics, 117 (2015) 17E502.

[13] L. Liu, T. Moriyama, D. C. Ralph, and R. A. Buhrman, Physical Review Letters, 106 (2011) 036601.

[14] K. Perzlmaier, M. Buess, C. H. Back, V. E. Demidov, B. Hillebrands, and S. O. Demokritov, Physical Review Letters, 94 (2005) 057202.

[15] X. J. Luo, P. H. Zhou, X. Wang, J. L. Xie, and L. J. Deng, Magnetics, IEEE Transactions on, 51(11) (2015)1-4.

[16] D. Kirk, A. Kohn, K. B. Borisenko, C. Lang, J. Schmalhorst, G. Reiss, and D. J. H. Cockayne, Physical Review B, 79 (2009) 014203.

[17] K. Mohri, F. B. Humphrey, K. Kawashima, K. Kimura, and M. Mizutani, Magnetics, IEEE Transactions on, 26(5) (1990) 1789-1791.

[18] S. Mallick, S. Bedanta, Journal of Magnetism and Magnetic Materials, 382 (2015) 158-164.

[19] T. Liu, P. H. Zhou, D. F. Liang, J. L. Xie, and L. J. Deng, Journal of Alloys and Compounds, 524 (2012) 1-4.

[20] T. M. Guo, J Magnetic Materials Devices, 23 (1992) 22-27.

[21] L. Zhang, W. B. Zhu, H. Y. Zheng, X. Wang, M. Bi, N. Wang, ... and L. J. Deng, Magnetics, IEEE Transactions on, 51(11) (2015) 1-4. 